\title{
Planetary Survival and Ejection in Transient Multiple Star Systems
}

\author{
Zeyang Meng ${ }^{1}$, Ji-Wei Xie ${ }^{1,2}$ and Ji-Lin Zhou ${ }^{1}$ \\ ${ }^{1}$ Department of Astronomy \& Key Laboratory of Modern Astronomy and Astrophysics in \\ Ministry of Education, Nanjing University, 210093, China \\ email: mengzy1989@gmail.com \\ ${ }^{2}$ Department of Astronomy and Astrophysics \\ University of Toronto, Toronto, ON M5S 3H4, Canada \\ email: jwxie@astro.utoronto.ca
}

\begin{abstract}
Many planets have been detected in close binary stars with separation only $\sim 20$ AU. These discoveries challenge the current theory of planet formation because binary stars with such an close separation are thought to have strong perturbations and thus inhibit planet formation around them. To address this issue, another scenario had been suggested: the binary separation was wider enough for binary formation in early stages, but it shrank to the present one after a transient triple star phase (stellar scattering phase). Here, we investigate how could planet survive or be ejected under this scenario. We find that (1) the odds of planetary survival are significantly reduced if scatterings between planets and/or planetesimals are included (2) circumbinary planets/planetesimals could be readily formed during such a transient phase.
\end{abstract}

Keywords. Planet and satellites: formation; Methods: N-body simulations; Stars: binaries

\section{Introduction}

Among more than 800 detected exoplanets so far (http://exoplanet.eu/), 60 of them reside in binary systems (Roell et al. 2012). Although most of these planet-bearing stars are wide binaries with separations $>100 \mathrm{AU}$, there are five of them with binary separation only 20 AU, including Gl86 Ab (Queloz et al. 2000), $\gamma$ Cephei Ab (Hatzes et al. 2003), HD41004 Ab (Zucker et al. 2004), HD196885 Ab (Chauvin et al. 2011), and most recently $\alpha$ Centauri Bb (Dumusque et al. 2012). Planets in such binary systems challenge the current planetary formation theory, because stars in such close separation induce strong perturbations and thus inhibit planet formation around them (Thébault et al. 2006; Thébault et al. 2008; Paardekooper et al. 2008; Xie \& Zhou 2008, 2009; Haghighipour 2010; Fragner et al. 2011; Giuppone et al. 2011).

The challenge becomes most critical in the case of HD196885 Ab, which is a giant planet with at least 3 Jupiter masses orbiting at 2.6 AU from the primary star. Given the orbit of the host binary (semimajor axis $a_{B}=21 \mathrm{AU}$ and eccentricity $e_{B}=0.42$ ), the planet is located very close to the boundary of stability (Holman \& Wiegert 1999), where is highly perturbed and therefore strongly hostile to planet formation (Thébault 2011. One of the solutions, as suggested by Thébault 2011, is that the binary had a initially wider orbit, but later shrunk to the present one via close stellar encounter. Such a kind of scenario was first (Pfahl 2005; Portegies \& McMillan 2005) applied to explain the origin of a planet candidate in HD188753 (Konacki, 2005) $\dagger$ and later to $\gamma$ Cephei Ab by Marzari \& Barbieri (2007a, 2007b) and Martí \& Beaugé (2012).

In this communication, we numerically investigate how could planets survive or be ejected during such a stellar encounter process. We extend the model of Marzari \&

$\dagger$ Its existence was later questioned and is still not confirmed (Eggenberger et al. 2007.) 
Barbieri (2007a) by including other planetary objects, e.g., earth mass planets and/or planetesimals. Such a consideration allows us to study the effect of planet-planet scattering during stellar encounter phase. In the following, we describe our model and present some preliminary results.

\section{Methods}

Following Marzari \& Barbieri (2007a), we consider a transient triple star system with masses and orbits elements summarized in table 1. Around the primary star, we assume a gas giant planet formed with 3 Jupiter mass at 2.6 AU (as HD196885 Ab) on a coplanar and circular orbit. In addition, between 0.5 and 2 AU round the primary, we put 1000 test particles (TPs, hereafter) aiming to model planetesimals or small planets. For each set of parameter shown in table 1 , we perform 100 simulations for $10^{7} \mathrm{yr}$ using the MERCURY software package (Chambers 1999) by choosing the BULIRSCH-STOER integrator.

Table 1. Characteristics of HD 196885 and Model Initial setups

\begin{tabular}{|c|c|c|c|c|c|}
\hline & Primary & $\begin{array}{c}\text { Star } \\
\text { Secondary }\end{array}$ & Tertiary & Gas Giant & TPs \\
\hline $\begin{array}{l}\text { HD } 196885 \\
\text { System }^{a}\end{array}$ & $m=1.3 \mathrm{M}_{\odot}$ & $\begin{array}{l}m=0.45 \mathrm{M}_{\odot} \\
a=21 \mathrm{AU} \\
e=0.42\end{array}$ & & $\begin{array}{l}m>3 \mathrm{M}_{\mathrm{J}} \\
a=2.6 \mathrm{AU} \\
e=0.48\end{array}$ & \\
\hline $\begin{array}{l}\text { Model } \\
\text { Initial } \\
\text { Setups }^{b}\end{array}$ & $m=1.0 \mathrm{M}_{\odot}$ & $\begin{array}{l}m=0.4 \mathrm{M}_{\odot} \\
a=35 \mathrm{AU} \\
e=0.2 \\
i=0^{\circ}\end{array}$ & $\begin{array}{l}m=0.4 \mathrm{M}_{\odot} \\
a=70 \mathrm{AU} \\
e=0.2 \\
i=20^{\circ}\end{array}$ & $\begin{array}{l}m=3 \mathrm{M}_{\mathrm{J}} \\
a=2.6 \mathrm{AU} \\
e=0.0 \\
i=0^{\circ}\end{array}$ & $\begin{array}{l}a \in[0.5 \mathrm{AU}, 2 \mathrm{AU}] \\
e=0.0 \\
i=0^{\circ}\end{array}$ \\
\hline
\end{tabular}

\footnotetext{
${ }^{a}$ Chauvin et al. 2011

${ }^{b}$ For the secondary, planet and TPs, their orbital elements are with respect to the primary star, while for the tertiary star, its orbital elements are with respect to the barycenter of the inner binary stars. All other angular elements are set randomly from a uniform distribution.
}

\section{Results}

We summarize our preliminary results as the following:

- For the stars, such a initial orbital condition is generally long-term unstable. Stars would have close encounters and scattering with each other, eventually forming a closer binary with the third star being ejected out. In our 100 runs, there 2, 45, 53 cases with the primary, secondary and territory star being the ejected star, respectively. All ejections occurred within less than $10^{6} \mathrm{yr}$ as shown in Fig.1. At this timescale, planet formation should be probably still ongoing.

- For the giant planet initial around the primary star, as can be seen from Fig.2, Although most of them were ejected, there are still about 20 in 100 cases where the planet survives at least $10^{7} \mathrm{yr}$, suggesting it is quite possible to form a planet-bearing binary system like HD 196885 under the scenario of a transient triple star system.

- For the TPs, the results depend on if the giant planet is included in the simulation. For the case without the giant planet, the chance of TP survival, as shown in Fig.1 and 2, decreases with the increase of their initial semi-major axis and the duration of the transient triple star system phase. These results are expected since a TP would be more unstable if place it closer to the perturber with longer time span. For the case with giant planet, there is no such anti-correlation between the star ejection time and the remaining $\mathrm{TP}$ number, and the $\mathrm{TP}$ survival probability is significantly reduced as 


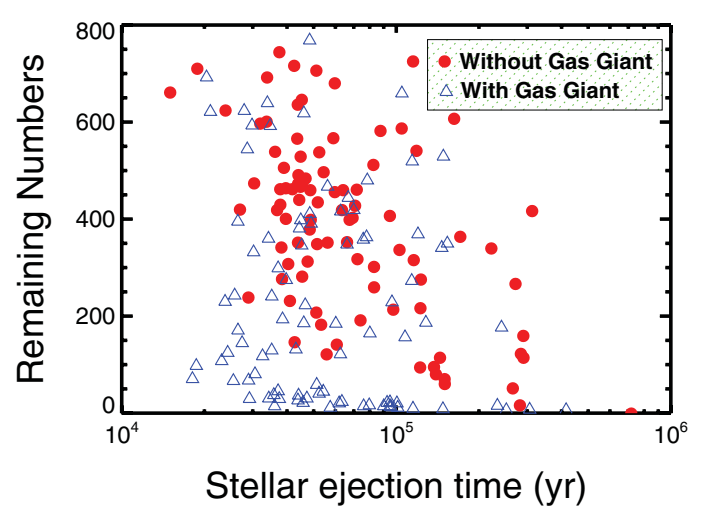

Figure 1. Number of remaining TPs as a function of time when the star is ejected, i.e., the duration of the transient triple star system phase. Triangles and circles are results of two sets of cases, respectively, with and without including the gas giant in the simulations. An anti-correlation between star ejection time and remaining TP number seems to appear in the latter.

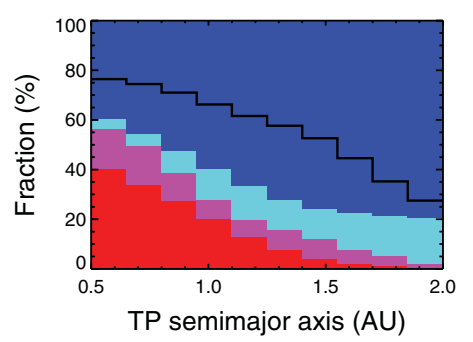

Figure 2. Possibilities of four types of outcomes $($ red $=$ giant ejection $+\mathrm{TP}$ survival, pink $=$ giant survival $+\mathrm{TP}$ survival, cyan $=$ giant survival $+\mathrm{TP}$ ejection and blue $=$ giant ejection + $\mathrm{TP}$ ejection). $\mathrm{X}$ axis denotes the initial semimajor axis of the TPs. Black histogram denote the results of without giant planet. TP survivals are much less if including the giant planet.

compared to the case without giant planet. Further more, in any case, for $>90$ out of 100 runs, we observed that some TPs were scattered onto orbits that finally around the later formed closer binary star, i.e., circumbinary TPs, suggesting that circumbinary planets/planetesimals should be common if stellar scattering occurred.

\section{References}

Chambers, John E. 1999, MNRAS, 304, 793

Chauvin, G.; Beust, H., Lagrange, A.-M., \& Eggenberger, A. 2011, A\&AA, 528, 8

Dumusque, X. et al. 2012, Nature, 491, 207

Eggenberger, A., Udry, S., Mazeh, T., Segal, Y., \& Mayor, M. 2007, A\& A, 466, 1179

Fragner, M. M., Nelson, R. P., \& Kley, W. 2011, A\&BA, 528, 40

Giuppone, C. A., Leiva, A. M., Correa-Otto, J., \& Beaugé, C. 2011, A\&\&A, 530, 103

Haghighipour, N. 2010, Planets in Binary Star Systems, ASSL, 366 (Springer)

Hatzes, A. P., Cochran, W. D., Endl, M., et al. 2003, ApJ, 599, 1383

Holman, M. J. \& Wiegert, P. A. 1999, AJ, 117, 621

Konacki, M. 2005, Nature, 436, 230

Martí, J. G. \& Beaugé, C. 2012, A\&̊A, 544, 97

Marzari, F. \& Barbieri, M. 2007, $A \mathscr{E} A, 467,347$

Marzari, F. \& Barbieri, M. 2007, A\&SA, 472, 643

Paardekooper, S.-J., Thébault, P., \& Mellema, G. 2008, A\& A, 386, 973

Pfahl, E. 2005, ApJ, 635, 89

Portegies Zwart, S. F. \& McMillan, Stephen L. W. 2005, ApJ, 633, 141

Queloz, D., Mayor, M., Weber, L., et al. 2000, A\&A, 354, 99

Roell, T., Neuhäuser, R., Seifahrt, A., \& Mugrauer, M. 2012, A\& $A, 542,92$

Schneider, J., Dedieu, C., Le Sidaner, P., Savalle, R., \& Zolotukhin, I. 2011, A\&SA, 532, 79

Thébault, P., Marzari, F., \& Scholl, H. 2006, Icar, 183, 193s

Thébault, P., Marzari, F., \& Scholl, H. 2008, MNRAS, 393, 21

Thébault, P. 2011, CeMDA, 111, 29

Xie, Ji-Wei \& Zhou, Ji-Lin 2008, ApJ, 686, 570

Xie, Ji-Wei \& Zhou, Ji-Lin 2009, ApJ, 698, 2066

Zucker, S., Mazeh, T., Santos, N. C., Udry, S., \& Mayor, M. 2004, A\&SA, 426, 695 